\title{
Efficient Extended Tree based Routing Protocol i.e. PSR for MANET
}

\author{
Supriya Kumari
}

\author{
Ankita Singh
}

\author{
Neetika Bhandari
}

\begin{abstract}
Routing algorithm have been proposed to solve node selection problem for forwarding the packets. Designing efficient routing approach for MANET is a challenging venture as here nodes have limited resource. Routing Protocols are categorized as Proactive, Reactive and Hybrid Protocols. Main challenges in MANET are that it needs dynamic routing which may results in frequent topological changes. In MANET routing overhead in terms of number of routing control messages must be minimized. In this paper we are trying to save the packets from being dropped by those nodes whose resource is going to be depleted in nearby future which will trim down the delay of messages and enhance packet delivery ratio. We have used NS2 tool and compared with basic routing algorithm.
\end{abstract}

\section{Keywords}

Mobile ad hoc networks (MANETs), Proactive routing, and Critical nodes.

\section{INTRODUCTION}

A Mobile ad hoc network is a wireless communication network. Here sender nodes which are not able to forward their messages to receiver nodes due to not being in the transmission range of each other will assign other node which is in transmission range of the sender node do the same. There are two most important Operations at the network layer, one is data forwarding and the other one is routing, but both are distinct concepts. Data forwarding checks how packets are taken from one link and put on another. Whereas function of routing is to determine which path a data packet should follow from the source node to the destination.

MANET protocols have to face high challenges due to dynamically changing of topologies, low transmission power and asymmetric links.[11] Due to link instability, node mobility and frequently changing topologies routing becomes one of the core issues in MANETs[11]

This paper presents a systematic evaluation of ETBP in comparison with two very well known protocols PSR and AODV. ETBP performs very well in terms of packet delay and packet drop in dynamic networks.

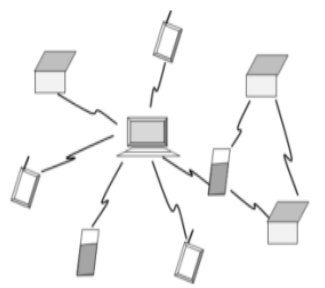

Fig 1: A Mobile Ad Hoc Network (MANET) [16]

\section{RELATED WORK}

Lots of routing protocols have been proposed till today for MANETs and it is concluded from them that providing a convenient routing protocol for MANETs is a difficult task due to its dynamic environment. Thus, the suitability of a particular routing protocol depends on many parameters such as, size of network, node mobility, and traffic load. All that together with the limited resources in MANETs (e.g. bandwidth, energy) makes the selection of an optimum routing protocol into a complicated task.

\subsection{Reactive routing protocol}

It is a bandwidth efficient on-demand routing protocol.

\subsubsection{Dynamic source routing}

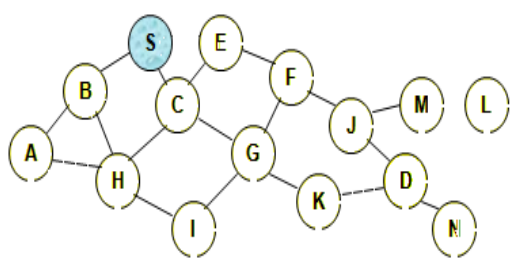

Fig.2. Nodes in DSR [9]

When node $\mathrm{S}$ wants to send a packet to node $\mathrm{D}$, but does not know a route to $\mathrm{D}$, node $\mathrm{S}$ starts a route discovery. $\mathrm{S}$ floods network with Route Request (RREQ). Route Reply (RREP) is sent by $\mathrm{D}$ on receiving the first RREQ. RREP includes the route from $\mathrm{S}$ to $\mathrm{D}$ on which RREQ was received by node $\mathrm{D}$ [12].

Its main advantage is that overhead of route maintenance is reduced and route discovery is reduced due to route cache. And limitation is that with route length, packet header size also increases due to source routing.

\subsubsection{Ad-hoc On-Demand Distance Vector \\ Routing $(A O D V)$}

It has both the advantages of DSR and DSDV protocol. AODV inherits the sequence numbers of DSDV and minimizes the amount of mute information flooding by creating routes on-demand, and improves the routing scalability and efficiency of DSR, which carries the source route in the data packet.[11] Here source broadcasts a RREQ(route request packet) to find route to the destination. Then a reply packet is sent by the destination or the intermediate node to the source along the same path through which packet arrived to destination. 


\subsection{Proactive routing protocol}

\subsubsection{Destination Sequenced Distance Vector protocol.}

Here each node maintains a list of all destinations and number of hops to each destination.

Its main advantage is that it solves the problem of loops while routing and count to infinity with help of sequence number. And limitation is that all nodes remain active, therefore no sleeping nodes and thus results in more power consumption and life of mobile node decreases. And other limitation is of overhead (most routing information never used, therefore unnecessary delay).

\subsection{Hybrid Routing Protocol}

\subsubsection{ZRP (zone routing protocol)}

It is a hybrid protocol of reactive and proactive routing protocol. ZRP can be classed as a hybrid reactive/proactive routing protocol. [16] Here it is assumed that there is huge amount of traffic around a node. Thus, ZRP reduces the proactive scope to a zone centered on each node. Hence it is easier to maintain routing information in a limited zone. Also reactive protocol can be utilized to reach the farther node.

\subsection{Extremely Opportunistic Routing (ExOR)}

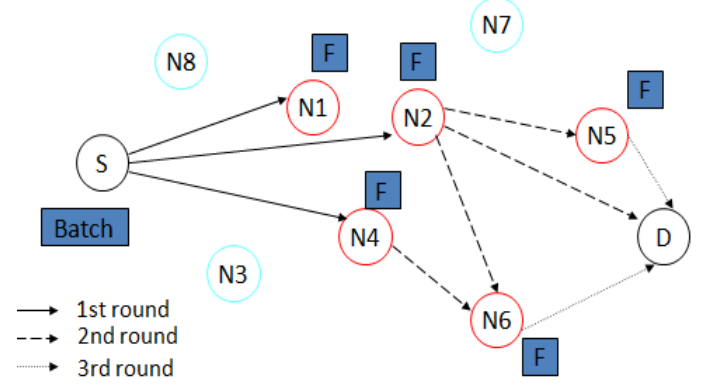

Fig 3: ExOR technique [2]

In [2] we can see that ExOR is a unicast routing technique for multi-hop wireless networks. Goal of ExOR is to improve throughput by minimizing number of retransmissions. Reason of retransmission is loss of packets being forwarded or it can be missing acknowledgments. To build forwarder map, source estimates best forwarding candidate and transmit a batch. Then acknowledgement is sent by the node based on the map which contains ID of the highest priority successful recipient. Decision is gossiped back to all stations with high probability which prevent collisions and thus forwarder transmits.

\section{Disadvantages}

- Packet delivery probability decreases with distance.

- Here overhead is introduced when header size increases along with batch size or packet is buffered in batches which effect latency and energy of mobile nodes in handling queued packets

Therefore ExOR in this form needs more refinement to route the packets for longer time with the limited energy of mobile nodes to serve as an attractive and deployable solution.

\section{PROBLEM DESCRIPTION}

As we know MANET have limited recourse in terms of battery power, bandwidth etc. And here position of node is changing continuously. So every time new route needs to be updated and thus it results in more consumption of battery power due to more no of packet retransmission (done for updating new route).

This in turn results in mobile node with very less remaining energy (battery power) which is not sufficient for forwarding of data packets. Here we call such node by a name called critical nodes.

And when a node failure occurs due to low energy, proactive routing protocols will have to wait until it receives fresh route information. This results in degraded performance in terms of packet drop which affects both packet delivery rate (which decreases) and packet delay (which increases).

Therefore we need an algorithm which deals with such circumstances where mobile nodes with very less energy are handled in such a way that, they are not selected for forwarding the packets.

\section{PROPOSED APPROACH}

As stated main problem with MANET is its limited resource and when a node transmits data packets, its energy gets consumed in this process. So packets should be transmitted in regulated manner such that packet drop is minimized. Packets are dropped in many situations. For example if there is congestion in a network then routers may drop the packets and inform sender about it. Packets can also be dropped by a mobile node if that node is dead or its energy decreases below threshold level. If packet drop is not controlled in efficient manner then it will affect both packet delivery ratio and time taken by packets to reach destination. If packet drop (by a node whose energy is below threshold level) is enhanced, then sender will have to retransmit those packets, which will lead to congestion in network. As a result delay of packets will increase. As for sending the same amount of messages more number of packets are actually transmitted (due to retransmissions), packet delivery ratio decreases.

So to deal with above problem we propose a method/solution which is as follows:

- Set the threshold value for power of a mobile node below which node becomes critical node.

- Critical node will alarm the sender that it will not be able to function in efficient manner soon .

- Sender will be informed about the status of remaining power of the critical node before it actually dies and stop forwarding packets.

- Sender will meanwhile select the next forwarder from the forwarding list which has next highest priority

- And as soon as sender gets the next forwarder, it stop sending packets to critical node, instead it now send packets to the selected forwarder.

Thus as here packets are not sent to the critical nodes, so chances of packets being dropped by this critical node is reduced by a great factor. As a result expected congestion will be reduced and thus packet delay and packet delivery ratio will not be affected. 


\subsection{Proposed algorithm/workflow}

\begin{tabular}{|l|l|l|l|}
\hline N2/P1 & N3/P2 & N4/P3 & N5/P4 \\
\hline
\end{tabular}

Fig 4: Forwarding list

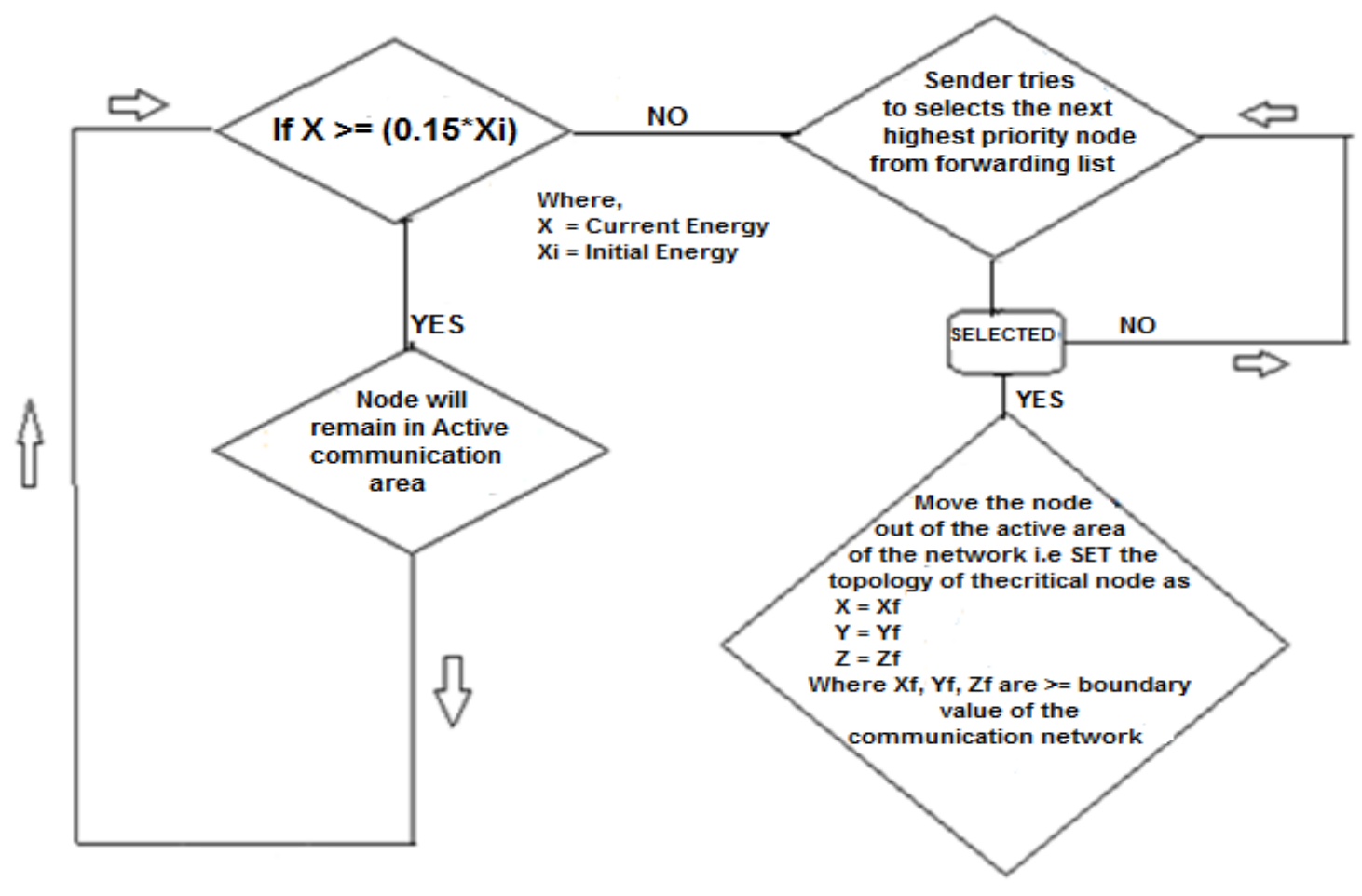

Fig 5: Flow chart representing proposed algorithm

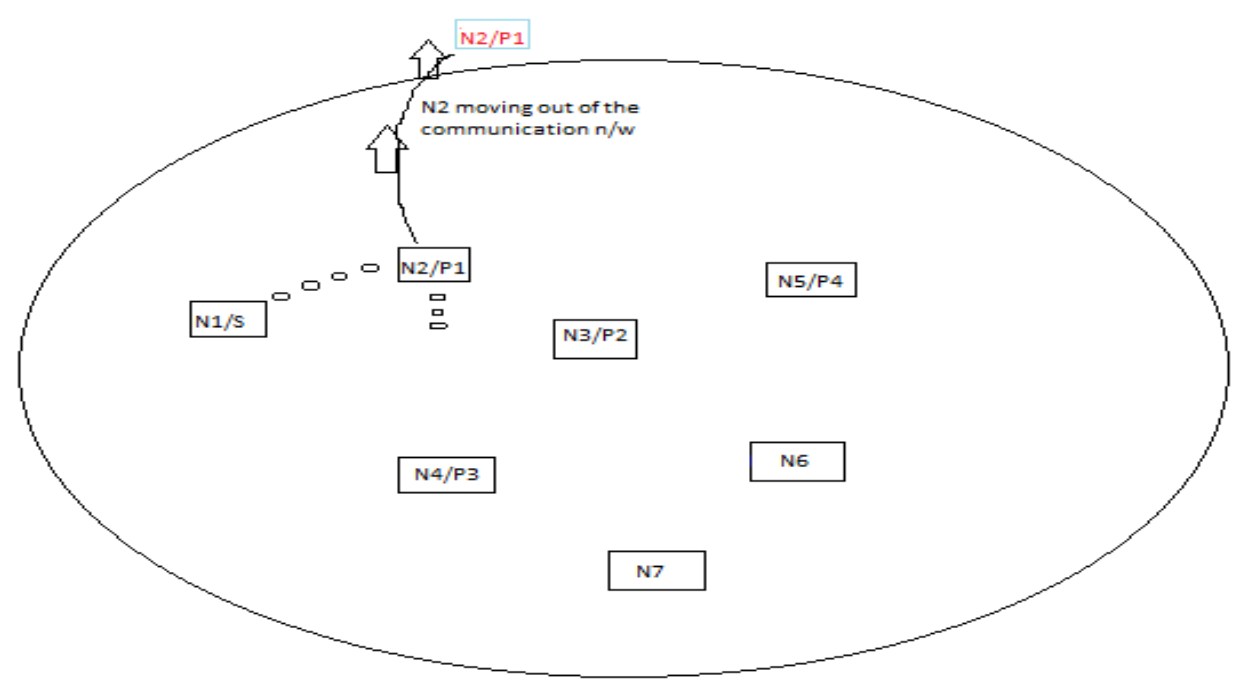

Fig.6: Nodes moving out of the active communication area

Here N2 is the critical node whose energy reaches below the threshold value. N1 will select the next best node according to the priority from forwarding list. Here N3 is selected as where Pi represent priority \& priority is as follows

$$
\mathrm{P} 1>\mathrm{P} 2>\mathrm{P} 3>\mathrm{P} 4
$$

Priority of mobile node is updated and maintained by sender and kept in forwarding list.

\section{to selects the next}

from forwarding list 
Let the ,

Initial energy of the node be $\mathrm{Xi}$

Threshold energy of node be $=15 \%$ of $\mathrm{Xi}$

Assumption :- And the initial energy will always be $100 \%$

$$
\text { If } x
$$$$
\text { If } \mathrm{X}>=(0.15 * \mathrm{Xi})
$$$$
\text { (intuitively) }
$$
\}

Then let the node be in the active area of the network Else

\{

Move the node out of the active area of the network

i.e SET the topology of the node as

$$
\mathrm{X}=\mathrm{Xf}
$$

$\mathrm{Y}=\mathrm{Yf}$

$Z=Z f$

Where Xf, Yf, Zf are $>=$ boundary value of the active communication area.

\}

\section{Fig 7: Proposed Algorithm}

\section{PROPOSED APPROACH}

The Proposed protocol is implemented in network simulator of version NS-2.35. In our simulation, 80 mobile nodes move for 150 seconds simulation time. We assume each node moves independently.Simulation parameters are summarized in table 1 .

Table 1. Simulation Criteria

\begin{tabular}{|c|c|}
\hline No of Nodes & 80 \\
\hline Area Size & $1000 \times 1000$ \\
\hline Simulation time & 150 sec \\
\hline
\end{tabular}

\section{Performance metrics}

\section{Packet delivery ratio}

Ratio of number of packets generated by source and number of packets delivered at the destination.

\section{Packet delay}

Time taken by packets to reach its destination.

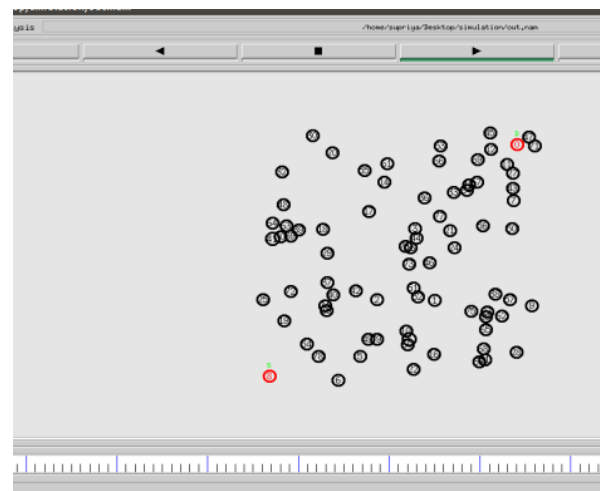

Fig 8: Initial Position of Nodes

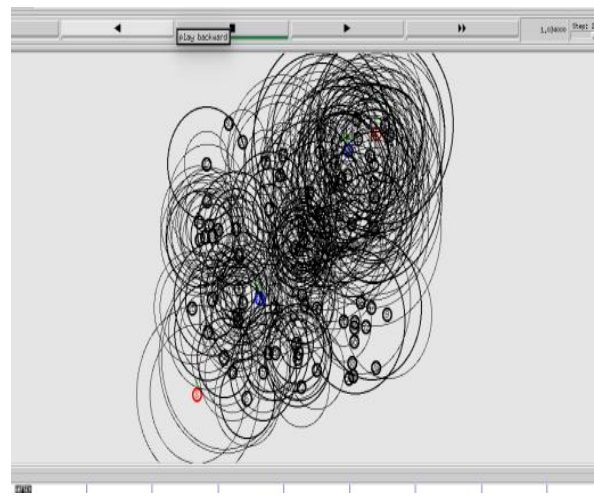

Fig 9: Traffic increased

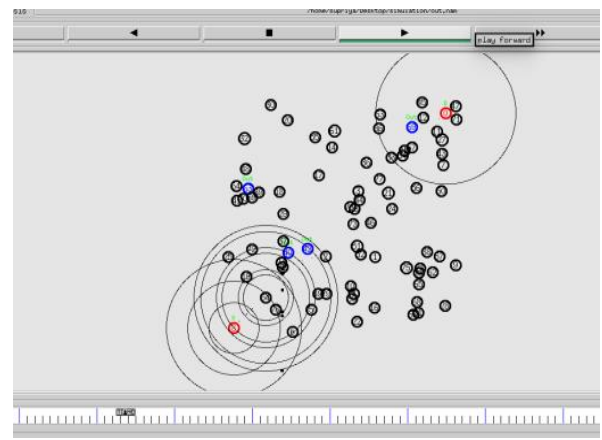

Fig 10: No of critical nodes increased and moving out of active area

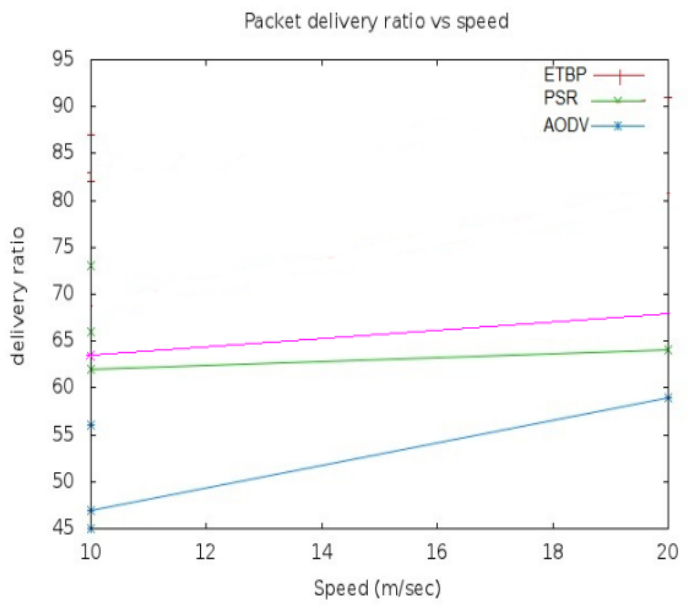

Fig 11: Packet delivery ratio vs speed

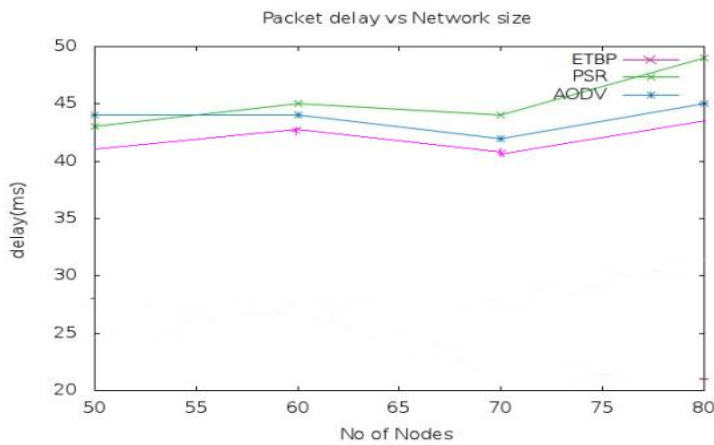

Fig 12: Packet delay verses number of nodes 


\section{CONCLUSION AND FUTURE WORK}

It is concluded that due to the random mobility of node, routing becomes a complex issue. Till now various routing protocols (each with distinct feature) are used in MANET. Based on the network environment's need suitable protocols are chosen. For example, Reactive routing protocols are suitable for such network where packet delay does not play a very important role. And this can be the case for non real time packet delivery. Whereas Proactive routing protocols are required for real time packets as all the information about the path are stored beforehand which results in huge space requirement storage of information which are stored in table. ETBP has been motivated by the need to support the dynamic nature of MANETs. ETBP can maintain more network topology information than most of the basic protocol at lesser space requirement. ETBP can handle the problem of packet delay and packet delivery ratio more efficiently as it can control the packets being dropped by the critical nodes by forwarding it to a non critical node. Thus now the number packets being retransmitted are reduced.

Here in ETBP two issues are dealt and they are packet delivery ratio and delay by considering the energy factor of mobile node. In future proposal we will try to use the same protocol for the secure transmissions of packets.

\section{REFERENCES}

[1] Zehua Wang, Yuanzhu Chen, Cheng Li, "CORMAN: A Novel Cooperative Opportunistic Routing Scheme in Mobile Ad Hoc Networks" in Communications, IEEE Journal on vol. 30, pp. 289-296, February 2012

[2] Biswas. S and R. Morris, "ExOR: Opportunistic MultiHop Routing for Wireless Networks," in Proc. ACM Conference of the Special Interest Group on Data Communication (SIGCOMM), Philadelphia, PA, USA, August 2005, pp. 133-144.

[3] C. E. Perkins and E. M. Royer, "Ad hoc On-Demand Distance Vector (AODV) Routing," RFC3561,July2003.[Online].

[4] Available:http://www.ietf.org/rfc/rfc3561.txtChlamtac. I, M. Conti, and J.-N. Liu, "Mobile Ad hoc Networking: Imperatives and Challenges," Ad Hoc Networks, vol. 1, no. 1, pp. 13-64, July 2003.

[5] C. Fragouli, J.-Y. L.Boudec, and J. Widmer, "Network Coding: an Instant Primer," SIGCOMM Computer
Communication Review, vol. 36, pp. 63-68, January 2006.

[6] Rajaraman.R, "Topology Control and Routing in Ad hoc Networks: A Survey," SIGACT News, vol. 33, pp. 6073, June 2002.

[7] SuhuaTANG , Bing ZHANG “A Robust AODV Protocol with Local Update " Adaptive communications research laboratories, Japan .

[8] Wang. Z, C. Li, and Y. Chen, "PSR: Proactive Source Routing in Mobile Ad Hoc Networks," in Proc. 2011 IEEE Conference Global Telecommunications (GLOBECOM), Houston, TX USA, December 2011.

[9] HumayunBakht, "Computing Unplugged, Wireless infrastructure, Some Applications of Mobile ad hoc networks", http://www.computingunplugged.com/issues/ issue200410/00001395001.html, April-2003.

[10] Loutfi, Valerie, Bruno. "Securing mobile adhoc networks", MP71 project, 2003

[11] Master Thesis Electrical Engineering Emphasis on Telecommunications Thesis no: MEE-2010-5834 May 2010 , Study and Performance Comparison of MANET Routing Protocols TORA, LDR and ZRP : Jia Uddin Md. Rabiul Zasad

[12] Mario Joa-Ng, “A Peer-to-Peer Zone-Based Two-Level Link State Routing for Mobile Ad Hoc Networks", IEEE Journal on selected areas in communications, Vol. 17, No.8, Aug-1999.

[13] PadminiMisra, "Routing Protocols for ad hoc mobile wireless Networks", http://www.cse.ohio-state.edu / jain/cis788-99/ftp/adhoc_routing/\#TDRP, Nov-1999.

[14] Sergio Marti, T. J. Giuli, Kevin Lai, Mary Baker"Mitigating Routing Misbehavior in Mobile Ad HocNetworks", Proceedings of the 6th annual international conference on Mobile computing and networking, Boston, Massachusetts, 2000,Pages: 255 265

[15] Young-BaeKo and Nitin H. Vaidya, "Location-Aided Routing (LAR) in mobile ad hoc networks", Proceedings of the 4th annual ACM/IEEE international conference onMobile computing and networking, Dallas, Texas, 1998, Pages: $66-75$ 